\title{
Vaginal Transitional Cell Metaplasia
}

National Cancer Institute

\section{Source}

National Cancer Institute. Vaginal Transitional Cell Metaplasia. NCI Thesaurus. Code C128062.

Vaginal metaplasia in which the vaginal surface squamous epithelium resembles benign transitional urothelium. 